RESEARCH ARTICLE

François Couchot

\title{
Finitistic weak dimension of commutative arithmetical rings
}

Received: 2 October 2010 / Accepted: 4 January 2011 / Published online: 24 March 2012

(C) The Author(s) 2012. This article is published with open access at Springerlink.com

\begin{abstract}
It is proven that each commutative arithmetical ring $R$ has a finitistic weak dimension $\leq 2$. More precisely, this dimension is 0 if $R$ is locally IF, 1 if $R$ is locally semicoherent and not IF, and 2 in the other cases.
\end{abstract}

Mathematics Subject Classification 13F05 - 13F30

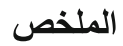

تم إثبات أن كل حلقة إبدالية حسابية R ذات بعد انتهائي ضعيف 2 2.

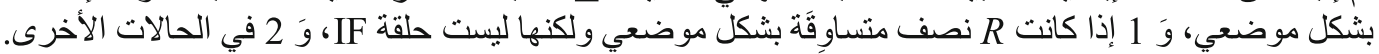

All rings in this paper are unitary and commutative. A ring $R$ is said to be a chain ring if its lattice of ideals is totally ordered by inclusion, and $R$ is called arithmetical if $R_{P}$ is a chain ring for each maximal ideal $P$. If $M$ is an $R$-module, we denote by w.d. $(M)$ its weak dimension. Recall that w.d. $(M) \leq n$ if $\operatorname{Tor}_{n+1}^{R}(M, N)=0$ for each $R$-module $N$. For any ring $R$, its global weak dimension w.gl.d $(R)$ is the supremum of w.d.( $M)$ where $M$ ranges over all (finitely presented cyclic) $R$-modules. Its finitistic weak dimension f.w.d. $(R)$ is the supremum of w.d. $(M)$ where $M$ ranges over all $R$-modules of finite weak dimension.

When $R$ is an arithmetical ring, by [9], a paper by Osofsky, we know that w.gl.d $(R) \leq 1$ if $R$ is reduced, and w.gl.d $(R)=\infty$ otherwise. By using the small finitistic dimension, similar results are proved by Glaz and Bazzoni when $R$ is a Gaussian ring satisfying one of the following two conditions:

- $R$ is locally coherent [7, Theorem 3.3];

- $\quad R$ contains a prime ideal $L$ such that $L R_{L}$ is non-zero and T-nilpotent (a slight generalization of [2, Theorem 6.4] using [1, Theorems $P$ and 6.3]).

They conjectured that these conditions can be removed. Recall that each arithmetical ring is Gaussian.

In this paper, we only investigate the finitistic weak dimension of arithmetical rings (it seems that it is more difficult for Gaussian rings). Main results are summarized in the following theorem:

Theorem 1 Let $R$ be an arithmetical ring. Then:

(1) f.w.d. $(R)=0$ if $R$ is locally $I F$;

(2) f.w.d. $(R)=1$ if $R$ is locally semicoherent and not locally IF;

F. Couchot $(\otimes)$

Laboratoire de Mathématiques Nicolas Oresme, Département de mathématiques et mécanique,

CNRS UMR 6139, 14032 Caen Cedex, France

E-mail: couchot@math.unicaen.fr 
(3) f.w.d.(R) $=2$ if $R$ is not locally semicoherent.

Let $\mathcal{P}$ be a ring property. We say that a ring $R$ is locally $\mathcal{P}$ if $R_{P}$ satisfies $\mathcal{P}$ for each maximal ideal $P$. As in [8], a ring $R$ is said to be semicoherent if $\operatorname{Hom}_{R}(E, F)$ is a submodule of a flat $R$-module for any pair of injective $R$-modules $E, F$. A ring $R$ is said to be IF (semi-regular in [8]) if each injective $R$-module is flat. If $R$ is a chain ring, we denote by $P$ its maximal ideal, $Z$ its subset of zerodivisors which is a prime ideal and $Q\left(=R_{Z}\right)$ its fraction ring. If $x$ is an element of a module $M$ over a ring $R$, we denote by $(0: x)$ the annihilator ideal of $x$ and by $\mathrm{E}(M)$ the injective hull of $M$.

Since flatness is a local module property, Theorem 1 is an immediate consequence of the following theorem that we will prove in the sequel.

Theorem 2 Let $R$ be a chain ring. Then:

(1) f.w.d.(R) $=0$ if $R$ is IF;

(2) f.w.d. $(R)=1$ if $R$ is semicoherent and not IF;

(3) f.w.d. $(R)=2$ if $R$ is not semicoherent. Moreover, an R-module $M$ has finite weak dimension if and only if $Z \otimes_{R} M$ is flat.

An exact sequence of $R$-modules $0 \rightarrow F \rightarrow E \rightarrow G \rightarrow 0$ is pure if it remains exact when tensoring it with any $R$-module. Then, we say that $F$ is a pure submodule of $E$. When $E$ is flat, it is well known that $G$ is flat if and only if $F$ is a pure submodule of $E$. An $R$-module $E$ is $F P$-injective if $\operatorname{Ext}_{R}^{1}(F, E)=0$ for any finitely presented $R$-module $F$. We recall that a module $E$ is FP-injective if and only if it is a pure submodule of every overmodule.

By [8, Proposition 3.3] the following proposition holds:

Proposition 3 A ring $R$ is IF if and only if it is coherent and self FP-injective.

The following proposition will be used frequently in the sequel.

Proposition 4 Let $R$ be a chain ring. The following conditions are equivalent:

(1) $R$ is semicoherent;

(2) $Q$ is an IF-ring;

(3) $Q$ is coherent;

(4) either $Z=0$ or $Z$ is not flat.

(5) for each non-zero element a of $Z \mathrm{E}(Q / Q a)$ is flat.

Proof By [5, Théorème 2.8] $Q$ is self FP-injective. So, (2) $\Leftrightarrow$ (3) by Proposition 3 and (1) $\Leftrightarrow$ (2) by [4, Corollary 5.2]. By applying [3, Theorem 10] to $Q$ we get that (2) $\Leftrightarrow(4) \Leftrightarrow$ (5).

The first assertion of Theorem 2 is an immediate consequence of the following proposition:

Proposition 5 For each IF-ring R, f.w.d. $(R)=0$.

Proof Let $0 \rightarrow F_{1} \rightarrow F_{0} \rightarrow K \rightarrow 0$ be an exact sequence where $F_{1}$ and $F_{0}$ are flat. Since $R$ is IF, $F_{1}$ is FP-injective (see [10, Lemma 4.1]). So, $F_{1}$ is a pure submodule of $F_{0}$. Consequently $K$ is flat. We deduce that each $R$-module $M$ satisfying $w . d .(M)<\infty$ is flat.

Lemma 6 Let $R$ be a chain ring. Then, for each non-zero element a of $P,(0: a)$ is a module over $Q$ and it is a flat $R /(a)$-module.

Proof Let $x \in(0: a)$ and $s$ a regular element of $R$. Since $R$ is a chain ring $x=s y$ for some $y \in R$, and $0=a x=s a y$. It follows that $a y=0$. Hence the multiplication by $s$ in $(0: a)$ is bijective.

If $c \in R$ we denote by $\bar{c}$ the coset $c+(a)$. Any element of $(\bar{c}) \otimes_{R /(a)}(0: a)$ is of the form $\bar{c} \otimes x$ where $x \in(0: a)$. Suppose that $c \notin(a)$ and $c x=0$. There exists $t \in R$ such that $a=c t \neq 0$. Since $R$ is a chain ring, from $c x=0$ and $c t \neq 0$ we deduce that $x=t y$ for some $y \in R$. We have $a y=c t y=c x=0$. So, $y \in(0: a)$ and $\bar{c} \otimes x=\bar{a} \otimes y=0$. Hence $(0: a)$ is flat over $R /(a)$.

Lemma 7 Let $p$ be an integer $\geq 1, R$ a chain ring and $M$ an $R$-module. Then w.d. $(M) \leq p$ if and only if $w . d .\left(M_{Z}\right) \leq p$. 
Proof Because of the flatness of $Q$, w.d. $(M) \leq p$ implies w.d. $\left(M_{Z}\right) \leq p$.

Conversely, let $0 \neq a \in R$. By Lemma $6(0: a)$ is a module over $Q$. So, from the exact sequences

$$
\begin{aligned}
& 0 \rightarrow(0: a) \rightarrow R \stackrel{a}{\rightarrow} R \rightarrow R /(a) \rightarrow 0, \\
& 0 \rightarrow(0: a) \rightarrow Q \stackrel{a}{\rightarrow} Q \rightarrow Q / Q a \rightarrow 0,
\end{aligned}
$$

we deduce for each integer $q \geq 1$ the following isomorphisms:

$$
\operatorname{Tor}_{q+2}^{R}(R /(a), M) \cong \operatorname{Tor}_{q}^{R}((0: a), M) \cong \operatorname{Tor}_{q}^{Q}\left((0: a), M_{Z}\right) \cong \operatorname{Tor}_{q+2}^{Q}\left(Q / Q a, M_{Z}\right) .
$$

So, if $p>1$ we get that w.d. $\left(M_{Z}\right) \leq p$ implies w.d. $(M) \leq p$. Now assume that $p=1$. Then, for each $a \in R$ we have:

$$
\operatorname{Tor}_{1}^{Q}\left(Q a, M_{Z}\right) \cong \operatorname{Tor}_{2}^{Q}\left(Q / Q a, M_{Z}\right)=0 .
$$

In the following commutative diagram

$$
\begin{aligned}
0 \rightarrow \operatorname{Tor}_{1}^{R}((a), M) & \rightarrow(0: a) \otimes_{R} M \rightarrow M \\
0 & \rightarrow(0: a) \otimes_{Q} M_{Z} \rightarrow M_{Z}
\end{aligned}
$$

the left vertical map is an isomorphism because $(0: a)$ is a $Q$-module (Lemma 6). It follows that the homomorphism $(0: a) \otimes_{R} M \rightarrow M$ is injective. We successively deduce that $\operatorname{Tor}_{1}^{R}((a), M)=0$ and $\operatorname{Tor}_{2}^{R}(R /(a), M)=0$. Hence w.d. $(M) \leq 1$.

From Lemma 7 we deduce the second assertion of Theorem 2:

Proof of Theorem 2(2) Assume that $R$ is semicoherent and not IF and let $M$ be an $R$-module with w.d. $(M)<$ $\infty$. Then we have w.d. $\left(M_{Z}\right)<\infty$. Since $Q$ is IF, $M_{Z}$ is flat by Proposition 5. By Lemma 7 w.d. $(M) \leq 1$. Moreover, since $R \neq Q$ then w.d. $(R /(a))=1$ for each $a \in P \backslash Z$. Consequently, f.w.d. $(R)=1$.

Lemma 8 Let $R$ be a chain ring which is not semicoherent. Then $Z \otimes_{R} M$ is flat for each $R$-module $M$ satisfying $w . d .(M) \leq 2$.

Proof Consider the following flat resolution of $M$ :

$$
0 \rightarrow F_{2} \rightarrow F_{1} \rightarrow F_{0} \rightarrow M \rightarrow 0 .
$$

If $a \in Z$ we have $\operatorname{Tor}_{p}^{R}((0: a), M) \cong \operatorname{Tor}_{p+2}^{R}(R /(a), M)=0$ for each integer $p \geq 1$. So, the following sequence is exact:

$$
0 \rightarrow(0: a) \otimes_{R} F_{2} \rightarrow(0: a) \otimes_{R} F_{1} \rightarrow(0: a) \otimes_{R} F_{0} \rightarrow(0: a) \otimes_{R} M \rightarrow 0 .
$$

By Lemma $6(0: a) \otimes_{R} F_{i}$ is flat over $R /(a)$ for $i=0,1,2$. By [3, Theorem 11(1)] $R /(a)$ is IF. We deduce that $(0: a) \otimes_{R} M$ is flat over $R /(a)$ by Proposition 5. If $K$ is the kernel of the map $F_{0} \rightarrow M$, it follows that the sequence $\mathcal{S}_{a}$

$$
0 \rightarrow(0: a) \otimes_{R} K \rightarrow(0: a) \otimes_{R} F_{0} \rightarrow(0: a) \otimes_{R} M \rightarrow 0
$$

is pure-exact over $R /(a)$, and over $R$ too. If $0 \neq r \in Z$, there exists $0 \neq a \in Z$, such that $r a=0$. Hence $Z=\cup_{a \in Z \backslash\{0\}}(0: a)$. So, if $\mathcal{S}$ is the sequence

$$
0 \rightarrow Z \otimes_{R} K \rightarrow Z \otimes_{R} F_{0} \rightarrow Z \otimes_{R} M \rightarrow 0,
$$

then $\mathcal{S}=\lim _{\rightarrow a \in Z \backslash\{0\}} \mathcal{S}_{a}$. By [6, Theorem I.8.13(a)] $\mathcal{S}$ is pure-exact. By Proposition $4 Z$ is flat. Consequently $Z \otimes_{R} F_{0}$ is flat and so is $Z \otimes_{R} M$.

Now, we can prove the third assertion of Theorem 2. 
Proof of Theorem 2(3) Assume that $R$ is not semicoherent. By [3, Proposition 14], the exact sequence

$$
0 \rightarrow Z \rightarrow Q \rightarrow Q / Z \rightarrow 0
$$

induces the following one for each non-zero element $a$ of $Z$ :

$$
0 \rightarrow Q / Z \rightarrow \mathrm{E}(Q / Z) \rightarrow \mathrm{E}(Q / a Q) \rightarrow 0 .
$$

From these two exact sequences we deduce the following one:

$$
0 \rightarrow Z \rightarrow Q \rightarrow \mathrm{E}(Q / Z) \rightarrow \mathrm{E}(Q / a Q) \rightarrow 0 .
$$

It is well known that $Q$ is flat. By [3, Proposition 8] $\mathrm{E}(Q / Z)$ is flat. Since $Q$ is not IF, $Z$ is flat and $\mathrm{E}(Q / a Q)$ is not flat by Proposition 4. So, f.w.d. $(R) \geq 2$. From the isomorphism (1) in the proof of Lemma 7 we deduce that f.w.d. $(R)=$ f.w.d. $(Q)$. So, we may assume that $R=Q$, hence the maximal ideal $P$ of $R$ is $Z$. By way of contradiction suppose that there exists an $R$-module $M$ with w.d. $(M) \geq 3$. We may replace $M$ with a syzygy module of a flat resolution and assume that w.d. $(M)=3$. We consider the following exact sequence where $F$ is flat:

$$
0 \rightarrow K \rightarrow F \rightarrow M \rightarrow 0 .
$$

Then w.d. $(K) \leq 2$. By Lemma $8 P \otimes_{R} K$ is flat. So, w.d. $\left(P \otimes_{R} M\right) \leq 1$ (recall that $P$ is flat by Proposition 4). Since w.d. $(R / P)=1$, then w.d. $(M / P M) \leq 1$ and w.d. $\left(\operatorname{Tor}_{1}^{R}(R / P, M)\right) \leq 1$ because these last modules are $R / P$-modules. We deduce from the exact sequence

$$
0 \rightarrow \operatorname{Tor}_{1}^{R}(R / P, M) \rightarrow P \otimes_{R} M \rightarrow P M \rightarrow 0
$$

that w.d. $(P M) \leq 2$ and from the following one

$$
0 \rightarrow P M \rightarrow M \rightarrow M / P M \rightarrow 0
$$

that w.d. $(M) \leq 2$. We get a contradiction. So, we conclude that f.w.d. $(R)=2$.

By Lemma 8 it remains to prove that w.d. $(M) \leq 2$ if $Z \otimes_{R} M$ is flat. As above we may assume that $R=Q$, and using again the exact sequences (2) and (3) we successively show that w.d. $(P M) \leq 2$ and w.d. $(M) \leq 2$.

If $A$ is a proper ideal of a chain ring $R$, let $A^{\sharp}=\{r \in R \mid A \subset(A: r)\}$. Then $A^{\sharp}$ is a prime ideal containing $A\left(A^{\sharp} / A\right.$ is the set of zerodivisors of $\left.R / A\right)$.

From Theorem 2 and [4, Corollary 5.3] we deduce the following:

Corollary 9 Let A be a non-zero proper ideal of a chain ring $R$. The following conditions are equivalent:

(1) $R / A$ is semicoherent;

(2) $A$ is either prime or the inverse image of a non-zero proper principal ideal of $R_{A^{\sharp}}$ by the natural map $R \rightarrow R_{A^{\sharp}}$;

(3) f.w.d.(R/A) $\leq 1$.

Using [3, Theorem 11] we deduce the following:

Corollary 10 Let $A$ be a non-zero proper ideal of a chain ring $R$. The following conditions are equivalent:

(1) $R / A$ is IF;

(2) either $A=P$ or $A$ is finitely generated;

(3) f.w.d.(R/A) $=0$.

From these corollaries we deduce the following examples:

Example 11 Let $R$ be a valuation domain which is not a field. Let $a$ be a non-zero element of its maximal ideal $P$. Then $R / a R$ is IF, so f.w.d. $(R / a R)=0$. Assume that $R$ contains an idempotent prime ideal $L \neq P$. If $a$ is a non-zero element of $L$, then $a L$ is a non-finitely generated ideal of $R_{L}$. So, $R / a L$ is not semicoherent and f.w.d. $(R / a L)=2$. It is well known that f.w.d. $(R)=$ w.gl.d $(R)=1$. Moreover, assume that $R$ contains a non-zero prime ideal $L \neq P$. Then, if $0 \neq a \in L$ then $a R_{L}$ is an ideal of $R$ and $R / a R_{L}$ is semicoherent and not IF. So, f.w.d. $\left(R / a R_{L}\right)=1$. 
Recall that a chain ring $R$ is strongly discrete if there is no non-zero idempotent prime ideal. From Theorem 1 and [4, Corollary 5.4] we deduce the following:

Corollary 12 Let $R$ be an arithmetical ring. The following conditions are equivalent:

(1) $R$ is locally strongly discrete;

(2) for each proper ideal $A, R / A$ is locally semicoherent;

(3) for each proper ideal A, f.w.d. $(R / A) \leq 1$.

Open Access This article is distributed under the terms of the Creative Commons Attribution License which permits any use, distribution, and reproduction in any medium, provided the original author(s) and the source are credited.

\section{References}

1. Bass, H.: Finitistic dimension and a homological generalization of semi-primary rings. Trans. Am. Math. Soc. 95, 466488 (1960)

2. Bazzoni, S.; Glaz, S.: Gaussian properties of total rings of quotients, J. Algebra 310, 180-193 (2007)

3. Couchot, F.: Injective modules and fp-injective modules over valuation rings. J. Algebra 267, 359-376 (2003)

4. Couchot, F.: Almost clean rings and arithmetical rings. In: Commutative Algebra and its Applications, pp 135-154. Walter de Gruyter, Berlin (2009)

5. Couchot, F.: Exemples d'anneaux auto fp-injectifs. Commun. Algebra 10(4), 339-360 (1982)

6. Fuchs, L.; Salce, L.: Modules Over Non-Noetherian Domains. Mathematical Surveys and Monographs, vol. 84. American Mathematical Society, Providence (2001)

7. Glaz, S.: Weak dimension of Gaussian rings. Proc. Am. Math. Soc. 133(9), 2507-2513 (2005)

8. Matlis, E.: Commutative semicoherent and semiregular rings. J. Algebra 95(2), 343-372 (1985)

9. Osofsky, B.: Global dimension of commutative rings with lineared ordered ideals. J. Lond. Math. Soc. 44, 183-185 (1969)

10. Stenström, B.: Coherent rings and FP-injective modules. J. Lond. Math. Soc. 2(2), 323-329 (1970) 